\title{
2. Non-Linear Theory of an Equatorial Flow. II
}

By Koji Hidaka, M. J. A.

Ocean Research Institute, Tokyo University, Tokyo

(Communicated Oct. 12, 1979)

Extension of the computation to higher latitudes. Once the velocity components $u$ and $v$ at the equator are known, the next question is how to compute those at higher latitudes. For this purpose, let us start with (1).

$$
2 \omega \sin \varphi v-v \frac{\partial u}{\partial y}=\frac{\partial D}{\partial x} ; \quad-2 \omega \sin \varphi u-v \frac{\partial v}{\partial y}=\frac{\partial D}{\partial y}
$$

From the first equation, it follows $\partial u / \partial y=2 \omega \sin \varphi-(1 / v)(\partial D / \partial x)$. Integrating this equation from 0 to $y$, we have

$$
\begin{aligned}
u-u_{0} & =2 \omega \int_{0}^{y} \sin \frac{\eta}{R} d \eta-\int_{0}^{y}\left(\frac{1}{v} \frac{\partial D}{\partial x}\right)_{y=\eta} \cdot d \eta \\
& =2 \omega R\left(1-\cos \frac{y}{R}\right)-\int_{0}^{y}\left(\frac{1}{v} \frac{\partial D}{\partial x}\right)_{y=\eta} \cdot d \eta
\end{aligned}
$$

where $u_{0}$ is the value of $u$ at the equator and both $v$ and $\partial D / \partial x$ are functions of $y$ only.

From the second equation of (1), it follows $(d / d y)\left(v^{2} / 2+D\right)$ $=-2 \omega \sin \varphi u$. Substituting from (2), it follows

$$
\begin{aligned}
& \frac{d}{d y}\left(\frac{v^{2}}{2}+D\right) \\
& \quad=-2 \omega \sin \frac{y}{R}\left\{u_{0}+2 \omega R\left(1-\cos \frac{y}{R}\right)-\int_{0}^{y}\left(\frac{1}{v} \frac{\partial D}{\partial x}\right)_{y=\eta} \cdot d \eta\right\} .
\end{aligned}
$$

Integrating this equation with respect to $y$, it follows

$$
\begin{aligned}
\frac{v^{2}}{2}+D= & \left(\frac{v_{0}^{2}}{2}+D_{0}\right)-2 \omega R \cdot u_{0} \int_{0}^{y} \sin \frac{\eta}{R} d\left(\frac{\eta}{R}\right) \\
& -(2 \omega R)^{2} \cdot \int_{0}^{y} \sin \frac{\eta}{R}\left(1-\cos \frac{\eta}{R}\right) d\left(\frac{\eta}{R}\right) \\
& +2 \omega R \cdot \int_{0}^{y} \sin \frac{\eta}{R} \cdot\left\{\int_{0}^{y}\left(\frac{1}{v} \frac{\partial D}{\partial x}\right)_{y=s} \cdot d s\right\} d\left(\frac{\eta}{R}\right)
\end{aligned}
$$

where $v_{0}$ and $D$ are the values of $v$ and $D$ at the equator.

If we confine the integration limit within a few degrees from the equator and change the order of integration, (3) becomes

$$
\begin{aligned}
\frac{v^{2}}{2}+D= & \frac{v_{0}^{2}}{2}+D_{0}-2 \omega R u_{0} \cdot\left\{\frac{1}{2}\left(\frac{y}{R}\right)^{2}-\frac{1}{4 !}\left(\frac{y}{R}\right)^{4}+\frac{1}{6 !}\left(\frac{y}{R}\right)^{6}\right\} \\
& -(2 \omega R)^{2}\left\{\frac{1}{8}\left(\frac{y}{R}\right)^{4}-\frac{1}{48}\left(\frac{y}{R}\right)^{6}+\frac{1}{640}\left(\frac{y}{R}\right)^{8}\right\}
\end{aligned}
$$




$$
+2 \omega R \cdot \int_{0}^{y}\left(\frac{1}{v} \frac{\partial D}{\partial x}\right)_{y=s} \cdot\left(\cos \frac{s}{R}-\cos \frac{y}{R}\right) d s .
$$

This equation gives the meridional component $v$ of the current in terms of the horizontal distance of a point $y$ from the equator, and geopotential distance $D$ at the point $y$.

Suppose there are the observational data at the equator and the integral degrees of meridional arc, or at $0^{\circ}(y=0), 1^{\circ} \mathrm{N}(=+\Delta), 2^{\circ} \mathrm{N}$ $(y=+2 \Delta)$ and $3^{\circ} \mathrm{N}(y=+3 \Delta)$. Since the length $\Delta$ of one degree of meridional arc is $\Delta=\pi R / 180=1.112 \times 10^{7} \mathrm{~cm}$, it is necessary to compute the velocity component $v$ at the above latitudes, use being made of the corresponding values of $D$. Let the velocity component $v$ at $1^{\circ} \mathrm{N}, 2^{\circ} \mathrm{N}$ and $3^{\circ} \mathrm{N}$ be denoted by $v_{1}, v_{2}$ and $v_{3}$ respectively.

It is essential now to express the integrals in the right-hand members in $n+2$ terms of $v_{1}, v_{2}$ and $v_{3}$. For this purpose, it will be recommended to apply Newton-Côtes' mean value method. ${ }^{6)}$ In evaluating these integrals, there are several formulas resulting from the Newton-Côtes' rule, or

$$
\begin{aligned}
& \int_{0}^{\Delta} F(s) d s=\left\{\frac{1}{2} F(0)+\frac{1}{2} F(\Delta)\right\} \Delta \\
& \int_{0}^{2 \Delta} F(s) d s=\left\{\frac{1}{6} F(0)+\frac{4}{6} F(\Delta)+\frac{1}{6} F(2 \Delta)\right\} \cdot 2 \Delta \\
& \int_{0}^{3 \Delta} F(s) d s=\left\{\frac{1}{8} F(0)+\frac{3}{8} F(\Delta)+\frac{3}{8} F(2 \Delta)+\frac{1}{8} F(3 \Delta)\right\} \cdot 3 \Delta .
\end{aligned}
$$

The formula (5), gives the so-called trapezoidal rule, while (6) is the well-known Simpson's rule. The formula (7) gives a rule applicable to the interval $(0 \leqq s \leqq 34)$. The accuracies of (6) and (7) are nearly the same, but the trapezoidal rule which replaces the curve by a straight line is much less accurate than two other formulas. In order to remove this difficulty, it is essential to replace the right-hand member of (5) by a difference of the rules (6) and (7), or

$$
\begin{aligned}
\int_{0}^{\Delta} F(s) d s= & \int_{0}^{3 \Delta} F(s) d s-\int_{\Delta}^{3 \Delta} F(s) d s \\
= & \left\{\frac{1}{8} F(0)+\frac{3}{8} F(\Delta)+\frac{3}{8} F(2 \Delta)+\frac{1}{8} F(3 \Delta)\right\} \cdot 3 \Delta \\
& -\left\{\frac{1}{6} F(\Delta)+\frac{4}{6}(2 \Delta)+\frac{1}{6} F(3 \Delta)\right\} \cdot 2 \Delta \\
= & \left\{\frac{3}{8} F(0)+\frac{19}{24} F(\Delta)-\frac{5}{24} F(2 \Delta)+\frac{1}{24} F(3 \Delta)\right\} \cdot \Delta .
\end{aligned}
$$

Applying the rules (8), (6) and (7) to the integrals at the righthand members of (3), we have after necessary numerical computations,

$$
v_{1}^{2}+Q_{1}=\frac{a_{12}}{v_{2}}+\frac{a_{13}}{v_{3}} ; \quad v_{2}^{2}+Q_{2}=\frac{a_{21}}{v_{1}} ; \quad v_{3}^{2}+Q_{3}=\frac{a_{31}}{v_{1}}+\frac{a_{32}}{v_{2}}
$$


where

$$
\begin{aligned}
Q_{1}= & -v_{0}^{2}+2\left(D_{1}-D_{0}\right)+28.2957 u_{0}+200.2734 \\
& -1180.26 \times 10^{5} \frac{(\partial D / \partial x)_{0}}{v_{0}}, \\
Q_{2}= & -v_{0}^{2}+2\left(D_{2}-D_{0}\right)+113.0793 u_{0}+3203.886 \\
& -4196.16 \times 10^{5} \frac{(\partial D / \partial x)_{0}}{v_{0}}, \\
Q_{3}= & -v_{0}^{2}+2\left(D_{3}-D_{0}\right)+254.0404 u_{0}+16215.556 \\
& -10620.62 \times 10^{5} \frac{(\partial D / \partial x)_{0}}{v_{0}},
\end{aligned}
$$

and

$$
\begin{array}{ll}
a_{12}=1966.90 \times 10^{5}\left(\frac{\partial D}{\partial x}\right)_{2}, & a_{13}=-1048.89 \times 10^{5}\left(\frac{\partial D}{\partial x}\right)_{3}, \\
a_{21}=12588.16 \times 10^{5}\left(\frac{\partial D}{\partial x}\right)_{1}, & a_{31}=28320.00 \times 10^{5}\left(\frac{\partial D}{\partial x}\right)_{1}, \\
a_{32}=17698.74 \times 10^{5}\left(\frac{\partial D}{\partial x}\right)_{2} . &
\end{array}
$$

It is possible to eliminate $v_{1}$ and $v_{2}$ from the three equations in (9). First it follows from the second equation,

$$
v_{1}=\frac{a_{21}}{v_{2}^{2}+Q_{2}} \text {. }
$$

Substituting this equation in the first equation of (9), it follows

$$
v_{3}=\frac{a_{12}}{\left(a_{21} /\left(v_{2}^{2}+Q_{2}\right)\right)^{2}+Q_{1}-a_{12} / v_{2}} \text {. }
$$
follows

Substituting (12) and (13) in the third equation of (9), it finally

or

$$
\frac{a_{12}^{2}}{\left\{\left(a_{21} /\left(v_{2}^{2}+Q_{2}\right)\right)^{2}+Q_{1}-a_{12} / v_{2}\right\}^{2}}+Q_{3}-\frac{a_{31}}{a_{21}}\left(v_{2}^{2}+Q_{2}\right)-\frac{a_{32}}{a_{2}}=0
$$

$$
\begin{aligned}
& \left\{Q_{3} v_{2}-\frac{a_{31}}{a_{21}} v_{2}\left(v_{2}^{2}+Q_{2}\right)-a_{32}\right\} \\
& \quad \times\left\{a_{21}^{2} v_{2}-a_{12}\left(v_{2}^{2}+Q_{2}\right)^{2}+Q_{1} v_{2}\left(v_{2}^{2}+Q_{2}\right)^{2}\right\}^{2}+a_{12}^{2} v_{2}^{3}\left(v_{2}^{2}+Q_{2}\right)^{4}=0 .
\end{aligned}
$$

This is an equation of 13th order and determines the possible value of $v_{2}$, or the meridional velocity at $2^{\circ} \mathrm{N}$. Substituting the value of $v_{2}$ thus obtained in (12) and (13), $v_{1}$ and $v_{3}$ will follow, or the meridional velocities at $1^{\circ} \mathrm{N}$ and $3^{\circ} \mathrm{N}$ respectively.

A simpler way of solving (9) is to neglect the squares $v_{1}^{2}, v_{2}{ }^{2}$ and $v_{3}^{2}$ in the left-hand members of (9). Then, from the second equation, it follows $v_{1}=a_{21} / Q_{2}$, from the third equation $v_{2}=a_{32} /\left(Q_{3}-\left(a_{31} / a_{21}\right) Q_{2}\right)$. Again inserting this in the first equation of (9), it follows $v_{3}=a_{12} /\left(Q_{1}-a_{12} / a_{32}\left(Q_{3}-\left(a_{31} / a_{21}\right) Q_{2}\right)\right)$. These are approximate values. Inserting them in the left-hand members of (7) gives more accurate roots. Repetition of this process results in an accurate set of roots of (9). 
In order to obtain the values of $u_{1}$ and $u_{2}$, it is enough only to transform the second equation of (1) as

$$
u=-\frac{(d / d y)\left(v^{2} / 2+D\right)}{2 \omega \sin \varphi}
$$

Since the quantity $v^{2} / 2+D$ is given for $1^{\circ}, 2^{\circ}$ and $3^{\circ} \mathrm{N}$, it follows from (16)

$$
u_{1}=-\frac{v_{2}^{2} / 2-v_{0}^{2} / 2+\left(D_{2}-D_{0}\right)}{2 \omega \sin 1^{\circ} \cdot 2 \Delta} ; \quad u_{2}=-\frac{v_{3}^{2} / 2-v_{1}^{2} / 2+\left(D_{3}-D_{1}\right)}{3 \omega \sin 2^{\circ} \cdot 2 \Delta} .
$$

In this process we cannot compute $u_{3}$, though we already have $v_{3}$ from (13). In the expressions (17), $v_{2}^{2} / 2-v_{0}^{2} / 2$ and $v_{3}^{2} / 2-v_{1}^{2} / 2$ are in most cases larger than $D_{2}-D_{0}$ and $D_{3}-D_{1}$ respectively. This is in very close agreement with $R$. B. Montgomery's conclusion on his dynamical computation which showed that the zonal component of velocity in the Equatorial Pacific is in an approximate geostrophic balance even in the $1^{\circ} \mathrm{N}$ intervals next to the equator. ${ }^{5)}$ This conclusion was also supported by J. A. Knauss (1960) who showed that the Coriolis force and the pressure gradient are in a balance in the northsouth direction except very near the equator.

For still higher latitudes $\left(|\varphi|>2^{\circ}\right)$, it is possible to compute these components by simpler successive approximations, transforming (1) into the form:

$$
u=\frac{-(\partial D / \partial y)-v(\partial v / v y)}{2 \omega \sin \varphi} ; \quad v=\frac{\partial D / \partial x+v(\partial u / \partial y)}{2 \omega \sin \varphi}
$$

with initial guesses $u=v=0$, using the values computed already at $2^{\circ} \mathrm{N}$ and $2^{\circ} \mathrm{S}$, and assuming that the flow at stations very far from the boundaries are purely geostrophic, or

$$
u=-\frac{\partial D / \partial y}{2 \omega \sin \varphi} ; \quad v=\frac{\partial D / \partial x}{2 \omega \sin \varphi} .
$$

This process fails at as low a latitude as $1^{\circ} \mathrm{N}$ and $1^{\circ} \mathrm{S}$ or in between, because the Coriolis parameter becomes too small. So we had better confine it for the latitudes higher than $3^{\circ} \mathrm{N}$ or $3^{\circ} \mathrm{S}$.

The result of practical application shows that at latitudes higher than $3^{\circ} \mathrm{N}$ or $3^{\circ} \mathrm{S}$, the flow is actually geostrophic.

Southern hemisphere. In order to compute $u$ and $v$ for the southern hemisphere, we have to solve

where

$$
\begin{aligned}
& v_{-1}^{2}+Q_{-1}=-\frac{a_{12}}{v_{-1}}-\frac{a_{13}}{v_{-3}} ; \quad v_{-2}^{2}+Q_{-2}=\frac{a_{21}}{v_{-1}} ; \\
& v_{-3}^{2}+Q_{-3}=-\frac{a_{31}}{v_{-1}}-\frac{a_{32}}{v_{-2}}
\end{aligned}
$$

$$
\begin{aligned}
Q_{-1}= & -v_{0}^{2}+2\left(D_{-1}-D_{0}\right)+28.2957 u_{0}+200.2734 \\
& +1180.26 \times 10^{5} \frac{(\partial D / \partial x)_{0}}{v_{0}}
\end{aligned}
$$




$$
\begin{aligned}
Q_{-2}= & -v_{0}^{2}+2\left(D_{-2}-D_{0}\right)+113.079 u_{0}+3203.886 \\
& +4196.16 \times 10^{5} \frac{(\partial D / \partial x)_{0}}{v_{0}}, \\
Q_{-3}= & -v_{0}^{2}+2\left(D_{-3}-D_{0}\right)+254.040 u_{0}+16215.556 \\
& +10620.62 \times 10^{5} \frac{(\partial D / \partial x)_{0}}{v_{0}},
\end{aligned}
$$

and $a_{12}, a_{13}, a_{21}, a_{31}$ and $a_{32}$ are the quantities given by (11).

The east-west components $u_{-1}$ and $u_{-2}$ can be computed by

$$
u_{-1}=\frac{v_{0}^{2} / 2-v_{-2}^{2} / 2+\left(D_{0}-D_{-2}\right)}{2 \omega \sin 1^{\circ} \cdot 2 \Delta} ; \quad u_{-2}=\frac{v_{-1}^{2} / 2-v_{-3}^{2} / 2+\left(D_{0}-D_{-3}\right)}{2 \omega \sin 2^{\circ} \cdot 2 \Delta} \text {. }
$$

This process gives the components at $1^{\circ} \mathrm{S}$ and $2^{\circ} \mathrm{S}$. For higher southern latitudes, successive approximation used for the northern latitudes will also apply without an alteration.

Upwelling velocity and the vertical circulation in a meridional section. The equation of continuity is, since $\partial u / \partial x=0$,

$$
\frac{1}{\cos \varphi} \frac{\partial}{\partial y}(v \cos \varphi)+\frac{\partial w}{\partial z}=0
$$

where $w$ is the vertical velocity counted positive downward. If this equation is integrated,

$$
w(z)-w(0)=-\frac{1}{\cos \varphi} \frac{\partial}{\partial y}\left(\cos \varphi \int_{0}^{z} v d z\right) .
$$

But, since there will be no vertical velocity at the sea surface $(z=0)$,

$$
w(z)=-\frac{1}{\cos \varphi} \frac{\partial}{\partial y}\left(\cos \varphi \int_{0}^{z} v d z\right)
$$

This is an expression of vertical component of velcoity in terms of $v$.

It is evident that the equation of continuity (46) will result in a stream function $\psi(y, z)$ such that

$$
v=\frac{\partial \psi}{\partial z} ; \quad \omega=-\frac{\partial \psi}{\partial y} .
$$

A constant value of the function $\psi$, of the equation:

$$
\psi(y, z)=\mathbf{a} \text { constant }
$$

gives stream lines of vertical circulation in a meridional section. The numerical values of stream function will be computed as

$$
\psi(y, z)=\cos \varphi \cdot \int_{0}^{z} v d z
$$

because there is a reason to suppose that the free sea surface is a stream line $\psi=0$.

Summary and conclusions. (1) A simple model was considered for the equatorial current system on the assumption that pressure gradients and current velocity do not change in the east-west direction. (2) A balance between the Coriolis forces, pressure gradients 
and field accelerations was assumed, $-w(\partial u / \partial z)$ and $-w(\partial v / \partial z)$ being neglected as small compared with $-v(\partial u / \partial y)$ and $-v(\partial v / \partial y)$ respectively. The friction due to mixing and thermodynamic process were disregarded. (3) The solution was carried out for a dynamic meridional section, first computing the velocity components at the equator, then extending calculation to higher latitudes of both hemispheres by different procedures. (4) An attempt was made to interpret the significance of Hidaka-Tsuchiya's formula of computing the east-west equatorial velocity. (5) The equatorial undercurrent can be located as an equatorial jet stream of a speed about $85 \mathrm{~cm} / \mathrm{sec}$ confind to a narrow band between $1^{\circ} \mathrm{S}$ to $1.5^{\circ} \mathrm{N}$. (6) The location and size of equatorial countercurrent agree with observations quite well. (7) It can be shown theoretically that the east-west component of velocity is approximately geostrophic even in the $1^{\circ}$ intervals next to the equator. (8) Upwelling velocities were determined and found to be an order of $-10^{-3} \mathrm{~cm} / \mathrm{sec}$. (9) Vertical circulation in a meridional section was determined by constructing the stream function between $v$ and $w$. (10) A brief discussion was made as to the merit of the inertia terms in hydrodynamical equations of an equational flow.

Acknowledgement. This research was made by the financial aid of the Japan Academy. The author is much indebted to Dr. K. Wadati, President of the Academy, for his cordial help.

\section{References}

1) Hidaka, K. (1980): Proc. Japan Acad., 56B, 1-6.

2) Montgomery, R. B. (1961): The Equatorial Undercurrent from Geostrophic calculation. Abstracts of Symposium Papers. Tenth Pacific Science Congress, Honolulu, Hawaii 1961, p. 342.

3) Runge, C., und H. König (1924): Numerisches Rechnen. Julius Springer, Berlin, p. 271. 